\section{BEST IN CLASS FOR INTERDENTAL DEVICE}

Philips' Sonicare AirFloss has been announced as one of the winners of the prestigious 2014 Pride Institute 'Best of Class' Technology Award in Dentistry. The awards recognise exemplary products and technologies that are rapidly redesigning approaches to patient care and practice management.

The Sonicare AirFloss is a hand-held electronic interdental cleaning device intended to make it easy for patients who do not floss to effectively, yet gently, remove plaque in hard-to-reach interproximal areas, reducing gingivitis and improving gum health.

Over 2 million AirFloss devices have been sold worldwide and it has also achieved accreditation from the British Dental Health Foundation.

For more information about Sonicare AirFloss visit www. sonicare.co.uk or telephone 0800 0567222

\title{
TRAINING CENTRE WILL TEACH FAST AND FIXED IMPLANTS
}

Bredent UK Ltd has chosen Dental Excellence Harewood in Leeds as the Centre of Excellence and UK Training Academy for its pioneering Sky Implant System.

Bredent UK, a subsidiary of German manufacturer Bredent $\mathrm{GmbH}$, will train worldwide dentists and technicians wishing to qualify for the advanced Sky 'fast and fixed' implant technique at Dental Excellence Harwood.

The Sky 'fast and fixed' concept allows patients to receive a full set of fixed teeth for a brand new smile in just one day, alleviating the need for complex and lengthy augmentation procedures. It provides an extremely natural substitute to real teeth and uses between four and six implants to provide a non-removable arch of teeth. The permanent fixture into the jaw bone means that they don't come loose or fall out and there is no need for denture adhesives or powders.

Dental Excellence Harewood has a team of highly experienced dentists and the $£ 2$ million centre can facilitate state of the art training through its live casting capabilities where delegates can watch a live procedure while discussing the technical details with another consultant.

Bredent UK will train approximately 350 dental professionals during the course of the next year at Dental Excellence Harewood.

Up and coming dates are 19/20 September 2014 and 30/31 January 2015. The course fee is $£ 695$ and includes hotel accommodation, meals, transfers, and all lectures. For more details and to book a place contact Bredent UK on 01246559599 or visit www. bredent.co.uk/harewood.

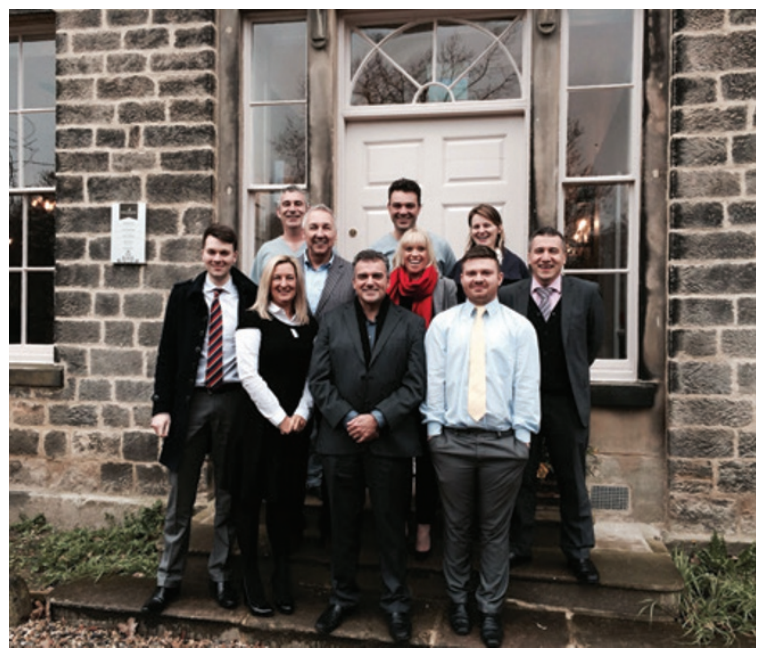

\section{INTEGRATED IMPLANTOLOGY AND SAFER IMPLANTS}

Sirona Dental Systems has introduced ground-breaking solutions for integrated implant planning and treatment. Solutions that combine the digital impression scanning and design capabilities of CEREC with the diagnostic accuracy of Sirona's 3D X-ray units.

Combine CEREC digital impressions and computer-aided design with the diagnostic security of Sirona's 3D X-ray systems for genuine integrated implantology. It is now possible to perform surgical and prosthetic planning simultaneously - and then implement this plan precisely using a surgical guide.

This is ideal for all practitioners who would like to place more implants in future - or those who want to provide implant therapy for the first time with higher clinical security as a result of the surgical guides and perfect documentation of the implant job. This caters for all stages of the implant process so you save time and money with fewer treatment sessions and achieve significant efficiency gains with a streamlined workflow. Last but not least, you can show your patients what the final result will look like, leading to higher treatment plan acceptance.

For further information call Sirona Dental Systems on 0845 0715040 or emailinfo@sironadental.co.uk. 